\title{
Pixels to Stitches: Embroidering Astronomy Images
}

\author{
Astronomers and artists recreate astronomical data with needle and \\ thread. \\ By Erika K. Carlson
}

I hile an emeritus professor at Harvard University in the 1970s, the astronomer Cecilia Payne-Gaposchkin received an unusual request: Would she embroider an x-ray image of the supernova remnant Cassiopeia A? A practitioner of needlepoint embroidery as well as of stellar spectroscopy, she obliged. Now, decades later, many astronomy researchers and enthusiasts are translating pixels of screen light to pixels of colored thread, rendering astronomical data and images in cross stitch, another form of embroidery.

Why is cross stitching especially well-suited to recreating

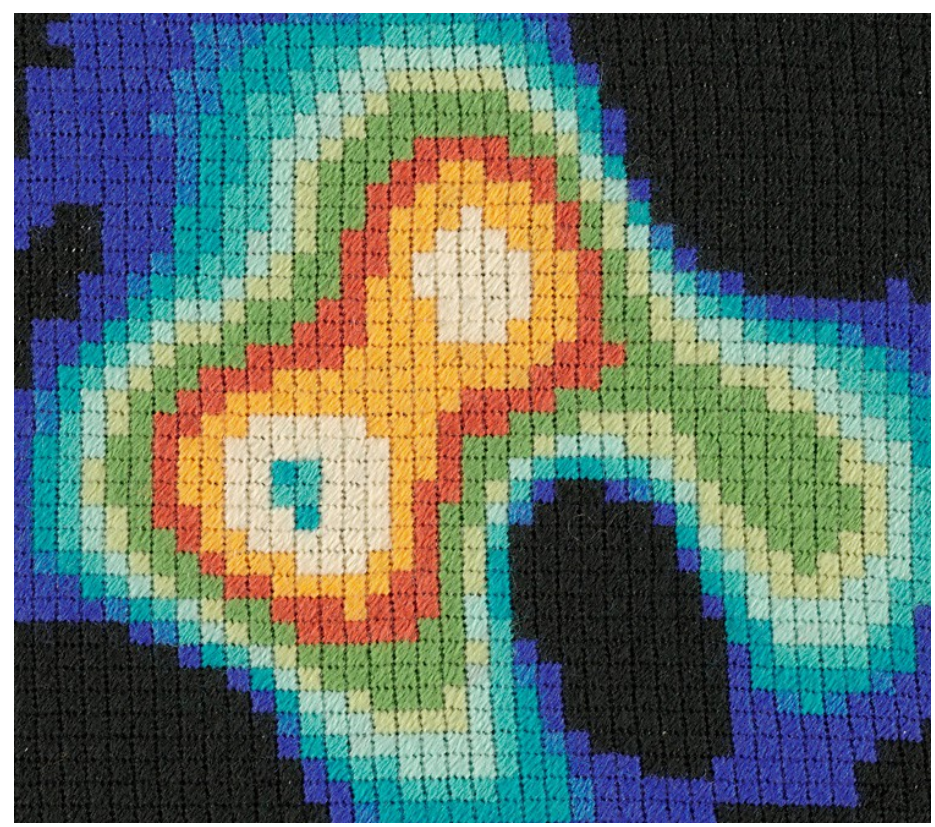

Needlepoint embroidery by the astronomer Cecilia

Payne-Gaposchkin of the supernova remnant Cassiopeia A.

Credit: Harvard University Archives, HUGB P182.XX astronomy data? "Basically, it's pixel art," says Yvette Cendes, an astronomer at the Center for Astrophysics, Massachusetts, and an avid cross stitcher. Cross-stitched designs consist of row after row of $x$-shaped stitches in a specialized fabric that has a grid of tiny holes (needlepoint designs are similar, but the stitches are different, and a type of canvas is typically used as the base fabric). To create designs, cross stitchers normally follow a cross-stitch pattern-a diagram that dictates which color of thread to use for any given square of the grid. Since astronomers often go through a similar process in presenting their data in 2D grids of pixels, cross stitch is a natural needle-and-thread cousin of astronomy.

Cendes has recreated a variety of astronomical data through cross stitch, from a radio image of a star being torn apart by a black hole to the Arecibo message that was broadcast into space in 1974. She has even stitched a reproduction of a figure from one of her papers-a 37-panel behemoth depicting snapshots of the famous supernova SN1987A's remains over a span of 25 years. She took great care in preserving the accuracy of the data she was stitching, including the color scale and coordinates. "It's the same information," Cendes says. "It's just in cloth instead of on your screen."

Like researchers fiddling with colors and axes on their plots, cross stitchers can control the look of their creations by adjusting several parameters. Thread colors offer an array of choices, of course, but changing the thickness of threads or the spacing of holes in the cloth can also affect how a piece looks.

"That's kind of what we do in astronomy, if you think about it," Cendes says. "We spend a lot of time trying to make the plot look pretty, right?" 


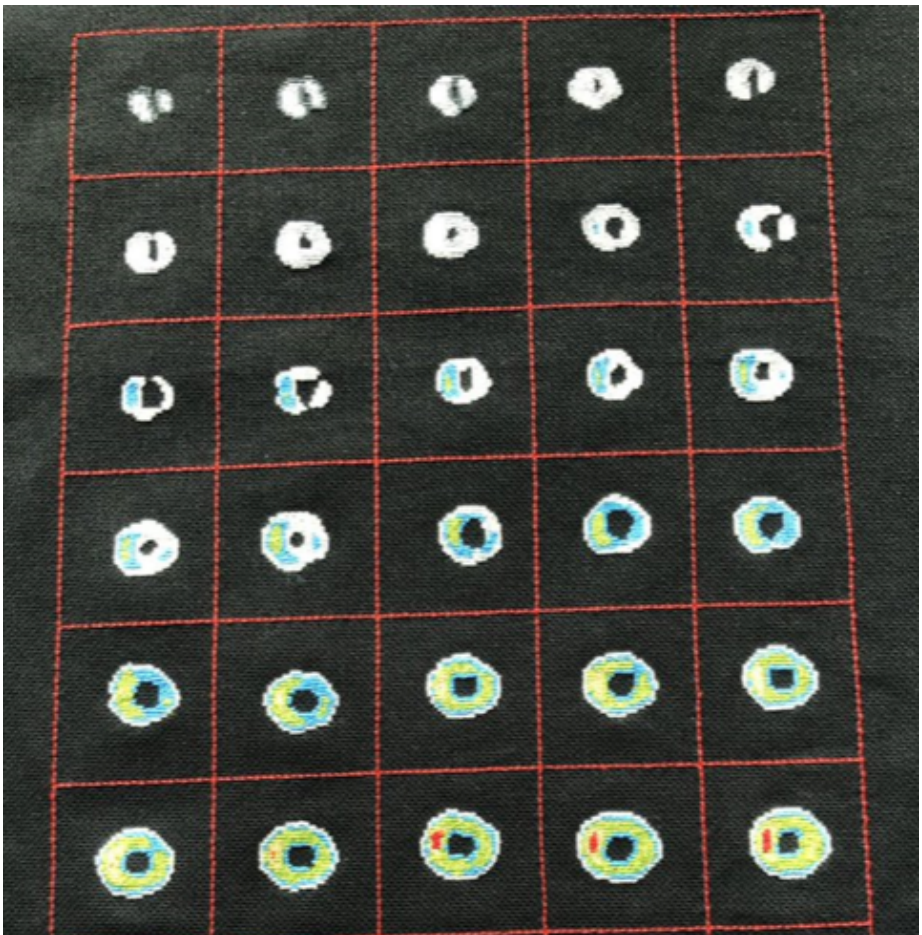

A series of cross-stitched panels by Yvette Cendes showing the time evolution of the supernova SN1987A, which was spotted in 1987 in a nearby galaxy.

Credit: Y. Cendes

Many other researchers have reproduced astronomical data in cross stitch. Mia de los Reyes, a graduate student in astronomy at Caltech, created a cross-stitch version of the supernova remnant $\mathbf{x}$-ray image that Payne-Gaposchkin rendered in needlepoint decades ago. Adi Foord, an astrophysicist at Stanford University, converted the Event Horizon Telescope's now-iconic black hole picture into a cross-stitch pattern, depicting the black hole's photon ring with a blaze of red, orange, and yellow threads on black cloth.

When it comes to extremely detailed astronomical images, such as photos of Solar System planets taken with the Hubble Space Telescope, translating them into manageable cross-stitch patterns can be challenging. Clare Bray, a cross stitcher in the UK who designs and sells cross-stitch templates in her online shop, bases many of her products on real astronomical images. To make a pattern of a celestial object, she uses a specialized computer program that allows her to draw the image and fill in
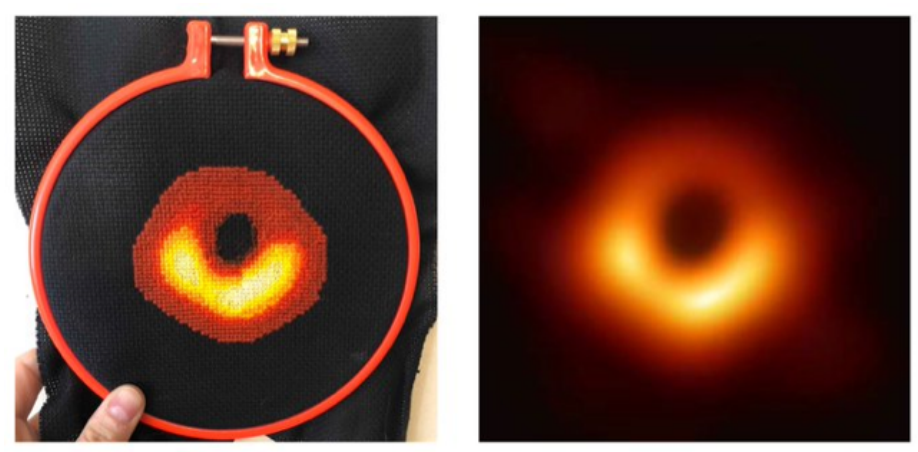

A cross-stitched representation by Adi Foord (left) of the image taken by radio astronomers of the supermassive black hole at the center of the galaxy M87 (right).

Credit: A. Foord/Event Horizon Telescope Collaboration

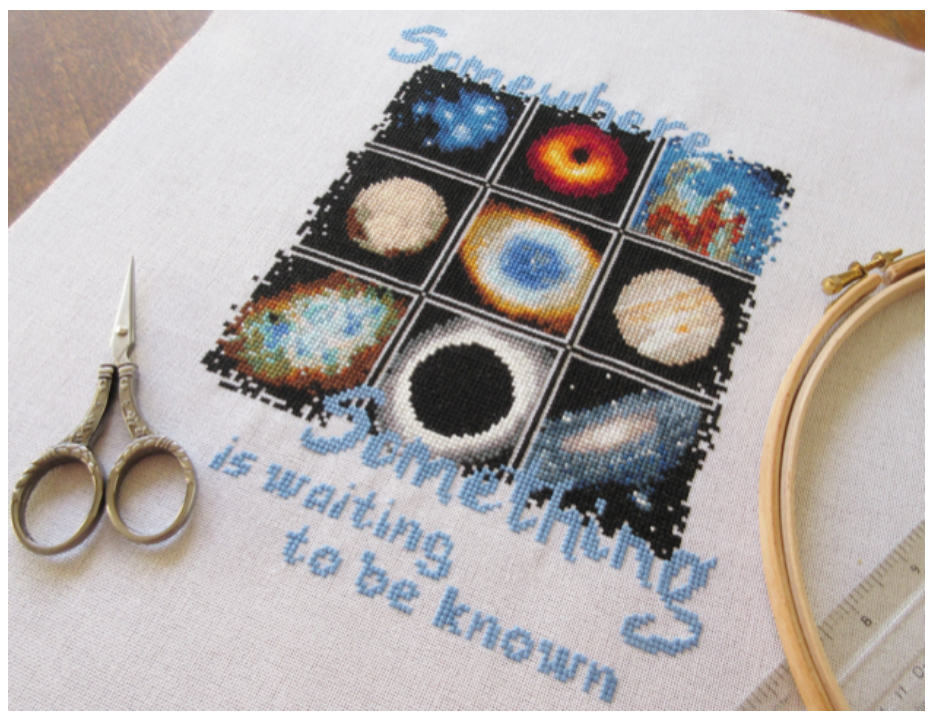

A montage of astronomical images designed by Clare Bay and stitched by Angie Sanders.

Credit: C. Bray

colors on a grid. She takes care to ensure that the stitched versions of astronomical observations have enough detail and complexity to be recognizable and attractive without requiring a daunting-and expensive-number of thread colors. "It's quite a balancing act," Bray says.

One of Bray's recent designs featured nine different astronomical objects as varied as Jupiter, the Crab Nebula, and 
the Andromeda Galaxy-all based closely on real images. She wondered if the montage would seem too serious, she says, but the design has been far more popular than she anticipated. She thinks people appreciated the specificity of the depictions-that it didn't include a generic drawing of a made-up galaxy, for example, but a faithful reproduction of an actual galaxy.

"I tend to be slightly literal when it comes to space," Bray says.

"I didn't think there was much point in making things up, because there's such great stuff out there anyway."

Bray, who has a bachelor's degree in physics, also wrote descriptions to go along with each of the featured objects in the design, including explanations of false color images and even some nuclear physics. Her customers range from cross-stitching research astronomers, including Cendes, to cross stitchers who know little about space but are drawn to the designs.

"You've really got the whole range, which has been really nice," Bray says. "This is for everyone."

Erika K. Carlson is a Corresponding Editor for Physics based in New York City. 